\title{
Knowledge, Experience, and Concerns Regarding Bed Bugs Among Emergency Medical Service Providers
}

\author{
Johnathan M. Sheele ${ }^{1}$, Osman Hamid ${ }^{1}$, Brandon F. Chang ${ }^{2}$, Jeffrey H. Luk ${ }^{2,} 3$ \\ 1. Emergency Medicine, Mayo Clinic, Jacksonville, USA 2. Emergency Medicine, University Hospitals Cleveland Medical \\ Center, Cleveland, USA 3. Emergency Medicine, Case Western Reserve University School of Medicine, Cleveland, USA
}

Corresponding author: Johnathan M. Sheele, sheele.johnathan@mayo.edu

\section{Abstract \\ Introduction}

Bed bugs are commonly encountered by emergency medical service (EMS) providers. The objective of this study was to determine the frequency with which EMS providers encountered bed bugs, assess their knowledge about bed bugs, and analyze the actions they take after finding bed bugs.

\section{Methods}

We anonymously surveyed 407 EMS providers from 180 EMS agencies in northeast Ohio between September 1, 2018, through March 31, 2019.

\section{Results}

Among the providers surveyed, $21 \%(n=84)$ of the EMS providers reported seeing bed bugs at least monthly, and $6 \%(n=24)$ reported seeing bed bugs at least weekly. Being younger, male, and working in an urban environment (vs. rural) were associated with EMS providers reporting more frequent bed bug encounters ( $\mathrm{p}$ : $\leqslant .05$ ). The mean level of concern for encountering bed bugs among EMS providers was 3.54 (SD: 1.15; scale: 1 $=$ no concern, 5 = very concerned). Among the EMS providers who reported seeing bed bugs at least monthly, $30 \%$ took the affected EMS stretcher out of service when they encounter a bed bug, $43 \%$ took the EMS rig out of service, $83 \%$ cleaned the EMS stretcher with a disinfectant, and $88 \%$ notified the ED that their patient has bed bugs. EMS providers scored poorly (mean: 69\% correct responses) in a seven-question assessment of basic bed bug biology and public health.

\section{Conclusion}

Based on our findings, we concluded that EMS knowledge and behavior related to bed bugs are suboptimal.

Received 04/06/2020

Review began 04/09/2020 Review ended 04/10/2020 Published 05/14/2020

(c) Copyright 2020

Sheele et al. This is an open access article distributed under the terms of the Creative Commons Attribution License CC-BY 4.0., which permits unrestricted use, distribution, and reproduction in any medium, provided the original author and source are credited.
Categories: Emergency Medicine, Infectious Disease, Environmental Health

Keywords: bed bug, bedbug, cimex lectularius, emergency medical service, ems, survey, epidemiology, risk factors, treatment, prehospital

\section{Introduction}

After near-eradication in the US, Cimex lectularius L. (C. lectularius), also known as the common bed bug, has been making a resurgence $[1,2]$. Bed bugs are now one of the most likely ectoparasites encountered by US healthcare providers [3-6]. In a 2018 survey, 59\% and 39\% of pest management professionals reported that they had decontaminated nursing homes and hospitals for bed bugs, respectively [7]. Emergency department (ED) visits related to bed bugs are estimated to have increased seven-fold between 2007 and 2010 [8].

C. lectularius is a hematophagous insect that preferentially feeds on humans $[1,9]$. The C. lectularius life cycle begins with an egg and proceeds through five nymphal stages before becoming an adult [1]. Each life stage requires a blood meal and a molt, and the insect can go from instar to adult in several weeks to months. $C$. lectularius feeds every few days but can go months without a blood meal [1,10]. Adult female C. lectularius lays an egg approximately every day when it has access to regular blood meals $[1,2]$. C. lectularius usually seeks refuge near their human host when not seeking a blood meal [1]. Most people develop an itchy rash after being fed upon by a bed bug, and bed bugs can be associated with human anxiety $[1,9,11]$. C. lectularius is not known to be an important vector of human infectious disease, although no major metagenomic investigation has examined its microbiome [1,12-16].

Evidence-based guidelines catering specifically to emergency medical service (EMS) providers on the management of bed bugs do not provide much information apart from generic suggestions about universal precautions, minimizing unnecessary exposure to infested patients, and preventing the spread of the insects 


\section{Cureus}

to uninfested persons $[1,2,17,18]$. There have been no studies exploring how EMS providers interact with bed bug-infested patients or assessing EMS provider knowledge about bed bugs. The objective of the study was to determine the frequency with which EMS providers encounter bed bugs, assess their knowledge of bed bugs, and analyze the actions they take after finding bed bugs.

\section{Materials And Methods}

Institutional review board approval was obtained from University Hospitals (UH) in Ohio to administer an anonymous survey to approximately 180 EMS agencies under UH medical command in northeast Ohio between September 1, 2018, and March 31, 2019. UH has an existing prehospital bed-bug policy of medical control procedures [19]. We eventually surveyed 407 EMS providers who were all $\geqslant 18$ years of age. Surveys with incomplete data were included in the final analysis. Only those respondents who reported seeing a bed bug on average every month or more frequently were included in the analyses of the 11 different potential actions that EMS providers could perform when they find a bed bug on a patient.

Continuous variables were analyzed using the t-test and analysis of variance (ANOVA). Categorical variables were evaluated with chi-square analysis. Linear and binomial logistic regression were performed accounting for age, gender (male vs. female), race (white vs. non-white), education (greater than or equal to a bachelor's degree vs. lower than a bachelor's degree), hours providing EMS patient care per week $(0-20 \mathrm{vs} \geqslant 20)$, level of training [greater than or equal to an emergency medical technician (EMT)-paramedic vs. lower than an EMTparamedic], the community the EMS provider predominantly serves (urban, rural, or suburban), if they have ever reported having a home bed bug infestation (yes vs. no), ever been fed upon by a bed bug (yes vs. no), ever seen a bed bug (yes vs. no), the number of years doing EMS ( $\leqslant 5,6-10,11-15$, or $>15$ years), knowledge of an existing bed bug protocol (yes, no, or unsure), the frequency with which EMS providers ask patients about bed bugs (yes vs. no), and the frequency with which EDs notify the EMS provider that they cared for a patient with bed bugs (yes vs no). Statistical significance was set at a p-value of $\leqslant .05$.

\section{Results}

The mean age of the survey respondents was 39.5 years (SD: 12.3 years); 89\% (361/404) of the respondents were white, and 93\% (376/406) were men. The respondent demographic characteristics and raw responses are summarized in Table 1.

\section{Characteristics}

Age, years, mean (SD)

Race $(n=404), n(\%)$

Non-white

White

$\operatorname{Sex}(n=406), n(\%)$

Female

Male

Education level $(n=406), n(\%)$

Trade/technical/vocational training

Some college credit, no degree

$130(32)$

Associate degree

Bachelor's degree

Master's or doctorate degree

Highest level of training, $n(\%)$

First responder

EMT-basic

$91(22)$

EMT-intermediate/advanced

$8(2)$

EMT-paramedic

$302(74)$

Hours/week transporting patients ( $n=405), n(\%)$ 


\section{Cureus}

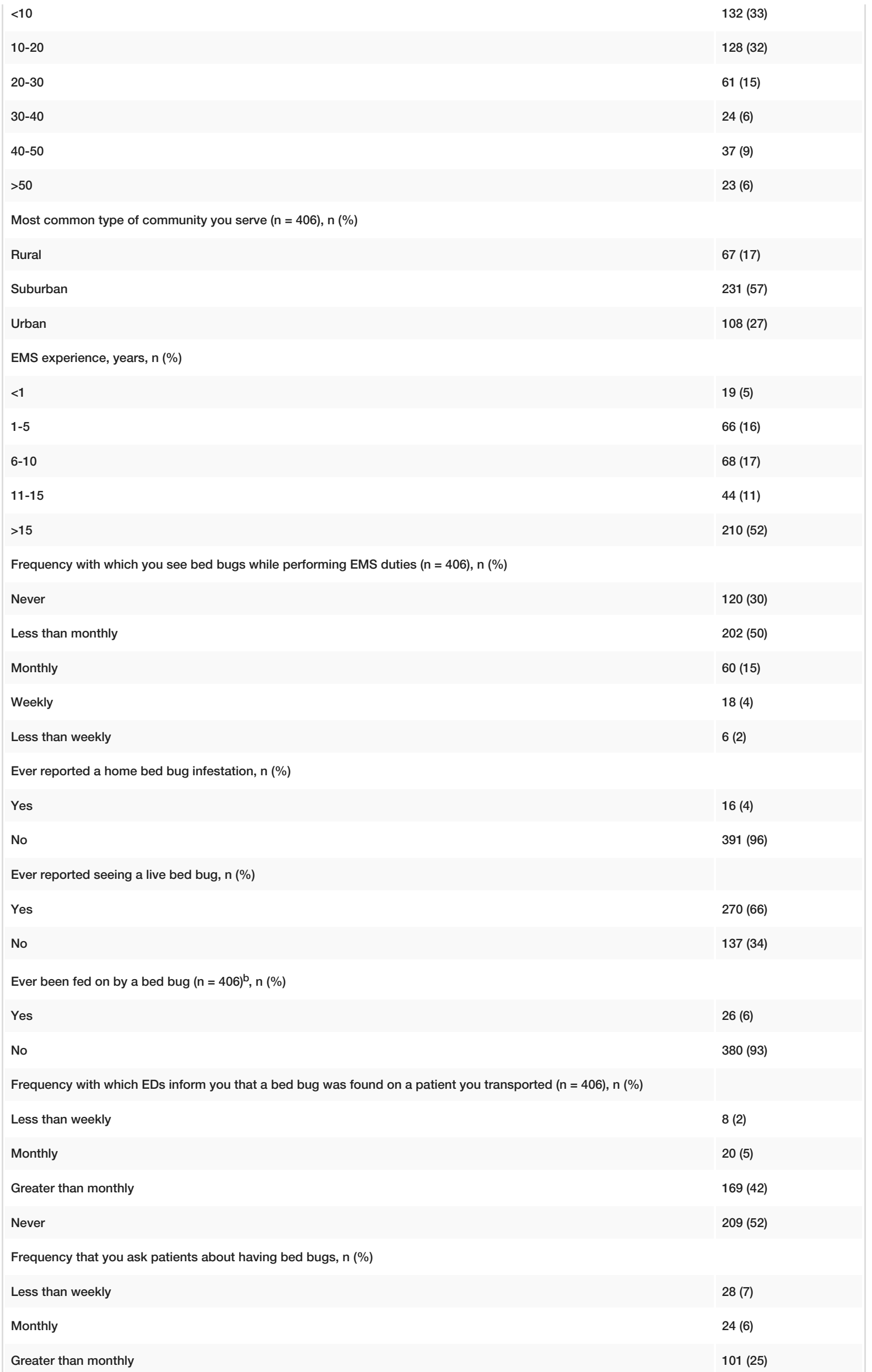




\section{Cureus}

Never

\section{TABLE 1: Demographic characteristics of survey respondents and summary of responses}

ED: emergency department; EMS: emergency medical services; EMT: emergency medical technician; SD: standard deviation

${ }^{a}$ Values are presented as number (percentage) of patients unless specified otherwise

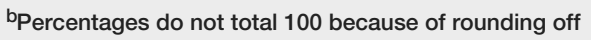

\section{Frequency of bed bug encounters}

Among the survey respondents, $21 \%(\mathrm{n}=84)$ of the EMS providers reported seeing bed bugs at least monthly, and $6 \%(n=24)$ reporting seeing bed bugs at least weekly. The EMS providers who reported seeing bed bugs at least weekly primarily worked in urban areas [50\% $(n=12)]$; while $46 \%(n=11)$ lived in the suburbs, and $4 \%(\mathrm{n}=1)$ lived in rural communities. By comparison, the 119 EMS providers who had never seen a bed bug were less likely to work in urban areas [urban: $15 \%(n=18)$; suburban: $56 \%(n=67)$; rural areas: $29 \%(n$ $=34) ; \mathrm{p}:<.001]$. EMT-paramedics were significantly more likely than non-EMT-paramedics to report seeing bed bugs weekly [83\% (20/24) vs. $17 \%$ (4/24); p: .002]. EMS providers who see bed bugs at least weekly were significantly more likely to ask their patients about bed bugs and be concerned about personally getting bed bugs ( $\mathrm{p}:<.001$ and $\mathrm{p}: .05$, respectively) compared to those EMS providers seeing bed bugs more than weekly. Also, $21 \%(n=5)$ of EMS providers who see bed bugs at least weekly also reported that the ED alerts them that they transported a patient with beds bugs at least weekly on average; while $17 \%(n=4)$ reported monthly, $21 \%(n=5)$ reported less than monthly, and $42 \%(n=4)$ never reported at all.

Among the respondents, 80\% (86/108) of predominantly urban EMS providers, $66 \%(152 / 231)$ of suburban providers, and $48 \%$ (32/67) of rural providers reported previously seeing a bed bug (p: <.001). Regression analysis identified the following EMS variables that were associated with a higher frequency of encountering bed bugs at work: being male, younger age, working in an urban rather than rural community, having previously seen a bed bug, more frequently asking patients about having home bed bugs, and more frequently reporting that an ED notified them that they transported a patient with bed bugs ( $\mathrm{p}: \leqslant .05$ for all) (Table 2). 


\section{Cureus}

\begin{tabular}{|c|c|c|c|c|}
\hline Variable & $\beta$ & $\mathbf{t}$ & SE (95\% Cl) & P-value \\
\hline Age, years & -0.01 & -1.98 & $0.005(-0.02$ to 0.07$)$ & . .05 \\
\hline \multicolumn{5}{|l|}{ Sex } \\
\hline Male vs. female & 0.26 & 2.08 & $0.12(0.01$ to 0.51$)$ & . .04 \\
\hline \multicolumn{5}{|l|}{ Race } \\
\hline White vs. non-white & 0.01 & 0.08 & $0.13(-0.24$ to 0.26$)$ & .94 \\
\hline \multicolumn{5}{|l|}{ Education level } \\
\hline Greater than or equal to a bachelor's degree vs. lower than a bachelor's degree & -0.05 & -0.56 & $0.09(-0.22$ to 0.12$)$ & .58 \\
\hline EMT-paramedic vs. non-EMT-paramedic & 0.06 & 0.69 & $0.09(-0.12$ to 0.25$)$ & . .49 \\
\hline Work in EMS $\geq 20$ vs. $0-20$, hours & 0.07 & 0.96 & $0.07(-0.07$ to 0.21$)$ & .34 \\
\hline \multicolumn{5}{|l|}{ Duration of working in EMS, years } \\
\hline $6-10$ vs. $11-15$ & -0.09 & -0.67 & $0.13(-0.34$ to 0.17$)$ & .50 \\
\hline$>15$ vs. $11-15$ & 0.04 & 0.30 & $0.13(-0.21$ to 0.28$)$ & .77 \\
\hline$\leq 5$ vs. $11-15$ & -0.10 & -0.75 & $0.14(-0.37$ to 0.17$)$ & 46 \\
\hline \multicolumn{5}{|l|}{ Frequency with which EMS provider asks the patient about bed bugs } \\
\hline Asks vs. never asks & 0.02 & 5.64 & $0.04(0.13$ to 0.28$)$ & \\
\hline \multicolumn{5}{|l|}{ Type of community the EMS serves } \\
\hline Suburban vs. rural & 0.02 & 0.24 & $0.09(-0.16$ to 0.21$)$ & .81 \\
\hline Urban vs. rural & 0.24 & 1.14 & 0.11 (0.03 to 0.45$)$ & . 03 \\
\hline \multicolumn{5}{|l|}{ Ever had a home bed bug infestation } \\
\hline Yes vs. no & -0.09 & -0.47 & $0.19(-0.47$ to 0.29$)$ & .64 \\
\hline \multicolumn{5}{|l|}{ Ever seen a bed bug } \\
\hline Yes vs. no & 0.84 & 10.68 & $0.08(0.68$ to 0.99$)$ & $<0.001$ \\
\hline Frequency with which the ED notifies you that you cared for a patient with bed bugs & 0.19 & 3.68 & $0.05(0.09$ to 0.30$)$ & $<0.001$ \\
\hline
\end{tabular}

\section{TABLE 2: Variables associated with the frequency of EMS providers encountering bed bugs}

EMS: emergency medical services; EMT: emergency medical technician; SE: standard error; Cl: confidence interval

\section{Concerns about getting bed bugs}

The mean level of concern about getting bed bugs among EMS providers was 3.54 (SD: 1.15) $(1=$ no concern and 5 = very concerned). The mean level of concern about getting bed bugs was 3.8 (SD: 1.2) for providers working predominantly in an urban environment, 3.4 (SD: 1.2) for those working predominantly in a suburban environment, and 3.6 (SD: 1.1) for those predominantly working in a rural environment (p: .02). The variables associated with increased EMS provider concern about getting bed bugs on linear regression were: working $\geqslant 20$ hours per week in patient care [beta: -0.50 (95\% CI: -0.26 to 0.74 ), p: <.001]; working in a suburban vs. rural environment [beta: -0.34 ( $95 \%$ CI: -0.66 to -0.02 ), p: 0.04 ], and asking patients more frequently about possible home bed bug infestations [beta: 0.16 ( $95 \%$ CI: 0.03 to 0.29 ), p: .02].

\section{Risk factors associated with EMS providers reporting a past home bed bug infestation}

Among the respondents, 4\% $(n=16)$ of EMS providers reported that they had a previous home bed bug infestation. Binomial logistic regression identified non-white race [OR: 11.93 (95\% CI: 1.97 to 72.33 ), p: .007 ] and a previous history of being fed upon by a bed bug [OR: 39.85 (95\% CI: 7.58 to 209.41), p: <.001] as risk factors for a home bed bug infestation. 


\section{Cureus}

\section{EMS provider knowledge about bed bugs}

The mean number of correct responses to seven common bed bug knowledge-based questions was 4.87 (SD: 1.39 ) with an overall correct response rate of $69 \%$ (Table 3).

\section{Question}

Do bed bugs transmit infectious diseases to humans?

Yes

No

Can bed bugs jump?

Yes

No

Can bed bugs fly?

Yes

No

Do bed bugs feed on human blood?

Yes

No

Do bed bugs lay eggs under a person's skin?

Yes

No

Do you think bed bugs live only in unsanitary conditions?

Yes

No

Are bed bug infestations easily treated with medication prescribed by a physician?

Yes

No

Correct responses, mean

\section{Response, \% (no. ratio)}

$39(157 / 401)$

$61(244 / 401)^{a}$

$69(279 / 406)$

$31(127 / 406)^{a}$

$5(22 / 405)$

$95(383 / 405)^{a}$

$71(287 / 405)^{a}$

$29(118 / 405)$

$25(102 / 406)$

$75(304 / 406)^{a}$

$18(72 / 407)$

$82(335 / 407)^{a}$

$28(115 / 405)$

$72(290 / 405)^{a}$

$69(1,970 / 2,835)$

TABLE 3: Summary of responses to questions relating to knowledge about bed bugs

no.: number

aAnswered correctly

The majority of EMS providers gave correct responses to questions except that $69 \%(279 / 406)$ incorrectly thought bed bugs could jump. Linear regression identified working in a suburban vs. rural environment [beta: 0.46 (95\% CI: 0.06 to 0.86)], working in an urban vs. rural environment [beta: 0.56 (95\% CI: 0.10 to 1.01)], and working in EMS $\leqslant 5$ years vs. $11-15$ years [beta: -0.64 (95\% CI -1.22 to -0.06$)$ ] as the variables associated with scoring higher on the bed bug knowledge assessment (p: <.05).

\section{EMS provider actions when encountering bed bugs}

The most common actions taken by $21 \%(n=84)$ of EMS providers who reported seeing on average $\geqslant 1$ bed bug per month was: using extra blankets (58\%); inspecting their own clothes for bed bugs (82\%); cleaning the EMS stretcher with disinfectant (83\%); notifying the ED about the patient having bed bugs (88\%) (Table 4). 


\section{Cureus}

\begin{tabular}{|c|c|}
\hline Action & Responses, $\mathbf{n}(\%)$ \\
\hline Do nothing & $2(2)$ \\
\hline Use double-glove & $10(12)$ \\
\hline Wear face mask & $7(7)$ \\
\hline Wear hair nets & $4(5)$ \\
\hline Use extra blankets & $49(58)$ \\
\hline Inspect own clothes for bed bugs & $69(82)$ \\
\hline Clean EMS stretcher with disinfectant & $70(83)$ \\
\hline Take EMS stretcher out of service & $25(30)$ \\
\hline Steam-clean EMS rig and/or stretcher & $18(21)$ \\
\hline Take EMS rig out of service & $36(43)$ \\
\hline Notify ED about the bed bug & $74(88)$ \\
\hline Hour the EMS rig is taken out of service for a bed bug: $<1$ hour; $1-8$ hours; $9-24$ hours; $>24$ hours & 42 (55); 32 (42); 1 (1); 2 (3) \\
\hline
\end{tabular}

TABLE 4: Frequency with which EMS providers perform certain actions when they identify bed bugs on patients they are transporting

EMS: emergency medical services; ED: emergency department

EMS rigs are taken out of service when a bed bug stays on them for more than an hour by $55 \%$ of respondents and by $42 \%$ when a bed bug stays on them for one to eight hours. Only $2 \%(n=2)$ of EMS providers reported taking no action when seeing bed bugs.

\section{Discussion}

To our knowledge, this is the first study to investigate EMS provider knowledge and behaviors related to bed bugs. Risk factors for EMS providers reporting more frequent exposure to bed bugs included younger age, male gender, and working in an urban environment. EMS providers reported a moderate concern about getting bed bugs (mean: 3.54, on a scale of 1-5), and their knowledge about bed bug public health and biology demonstrated opportunities for more education.

The study population involved EMS agencies in and around Cleveland, Ohio, which is one of the most bed bug-infested cities in the United States [20]. At UH Cleveland Medical Center (UHCMC), a bed bug is found in the facilities on average every 2.2 days and every three to six days in the ED; however, the true number is likely underreported [6,21-23]. A survey of UHCMC ED patients found that about one in 50 reported an active home bed bug infestation; 37\% (253/680) reported a past history of being fed on by a bed bug, and $59 \%$ (415/702) reported knowing someone other than persons living in their home having a bed bug infestation in the past five years $[4,5]$. UHCMC ED patients with bed bugs are more likely to be older, male, brought to the ED by EMS providers, and admitted to the hospital [3-5]. Only $18 \%(n=2)$ of the $11 \mathrm{ED}$ patients reporting active home bed bug infestations stated that they notified EMS providers about the infestation [4]. A failure to isolate bed bug-infested patients in the ED puts other healthcare providers and patients at risk for infestation.

Our study has several limitations. Most of our study participants were white, male EMT-paramedics, who worked in a suburban community that has a high number of community bed bug infestations. Most of our respondents have provided EMS services for more than 15 years. The risk to EMS providers for acquiring a home bed bug from work-related duties is difficult to quantify from a survey and remains unknown. EMS provider responses to the knowledge-based questions and their reported actions when caring for bed buginfested patients demonstrate opportunities for additional education and training.

\section{Conclusions}

EMS providers, especially those working in an urban environment, encounter bed bugs frequently during patient care. Even though EMS providers are concerned about getting bed bugs, they have inadequate knowledge about bed bugs and their effects on human health. Most EMS providers reported low compliance with basic interventions to limit the spread of bed bugs, including taking the EMS rig out of service, cleaning 


\section{Additional Information Disclosures}

Human subjects: Consent was obtained by all participants in this study. Institutional Review Board, University Hospitals Cleveland Medical Center issued approval STUDY20180361. This study was approved by the Institutional Review Board of University Hospitals Cleveland Medical Center with the approval number STUDY20180361. Animal subjects: All authors have confirmed that this study did not involve animal subjects or tissue. Conflicts of interest: In compliance with the ICMJE uniform disclosure form, all authors declare the following: Payment/services info: All authors have declared that no financial support was received from any organization for the submitted work. Financial relationships: All authors have declared that they have no financial relationships at present or within the previous three years with any organizations that might have an interest in the submitted work. Other relationships: All authors have declared that there are no other relationships or activities that could appear to have influenced the submitted work.

\section{Acknowledgements}

We thank Leonard Keller, MD, for his assistance on the project.

\section{References}

1. Doggett SL, Dwyer DE, Peñas PF, Russell RC: Bed bugs: clinical relevance and control options. Clin Microbiol Rev. 2012, 25:164-92. 10.1128/CMR.05015-11

2. Munoz-Price LS, Safdar N, Beier JC, Doggett SL: Bed bugs in healthcare settings. Infect Control Hosp Epidemiol. 2012, 33:1137-42. 10.1086/668029

3. Sheele JM, Gaines S, Maurer N, et al.: A survey of patients with bed bugs in the emergency department . Am J Emerg Med. 2017, 35:697-8. 10.1016/j.ajem.2016.12.076

4. Sheele JM, Crandall CJ, Chang BF, Arko BL, Dunn CT, Negrete A: Characteristics of bed bug infested patients in the emergency department. Emerg Med Int. 2019, 2019:8721829. 10.1155/2019/8721829

5. Sheele JM, Crandall CJ, Chang BF, Arko BL, Dunn CT, Negrete A: Risk factors for bed bugs among urban emergency department patients. J Community Health. 2019, 44:1061-8. 10.1007/s10900-019-00681-2

6. Totten V, Charbonneau H, Hoch W, Shah S, Sheele JM: The cost of decontaminating an ED after finding a bed bug: results from a single academic medical center. Am J Emerg Med. 2016, 34:649. 10.1016/j.ajem.2015.12.020

7. National Pest Management Association: PPMA survey highlights bed bug prevalence nationwide . (2018). Accessed: September 23, 2019: https://pestworldmag.npmapestworld.org/2018/05/31/ppma-surveyhighlights-bed-bug-prevalence-nationwide/.

8. Langley R, Mack K, Haileyesus T, Proescholdbell S, Annest JL: National estimates of noncanine bite and sting injuries treated in US hospital emergency departments, 2001-2010. Wilderness Environ Med. 2014, 25:14-23. 10.1016/j.wem.2013.08.007

9. Goddard J, deShazo R: Bed bugs (Cimex lectularius) and clinical consequences of their bites . JAMA. 2009, 301:1358-66. 10.1001/jama.2009.405

10. Reinhardt K, Isaac D, Naylor R: Estimating the feeding rate of the bedbug Cimex lectularius in an infested room: an inexpensive method and a case study. Med Vet Entomol. 2010, 24:46-54. 10.1111/j.13652915.2009.00847.x

11. Goddard J, de Shazo R: Psychological effects of bed bug attacks (Cimex lectularius L.) . Am J Med. 2012, 125:101-3. 10.1016/j.amjmed.2011.08.010

12. Angelakis E, Socolovschi C, Raoult D: Bartonella quintana in Cimex hemipterus, Rwanda . Am J Trop Med Hyg. 2013, 89:986-7. 10.4269/ajtmh.13-0182

13. Leulmi H, Bitam I, Berenger JM, et al.: Competence of Cimex lectularius bed bugs for the transmission of Bartonella quintana, the agent of trench fever. PLoS Negl Trop Dis. 2015, 9:e0003789. 10.1371/journal.pntd.0003789

14. Adelman ZN, Miller DM, Myles KM: Bed bugs and infectious disease: a case for the arboviruses . PLoS Pathog. 2013, 9:e1003462. 10.1371/journal.ppat.1003462

15. Delaunay P, Blanc V, Del Giudice P, Levy-Bencheton A, Chosidow O, Marty P, Brouqui P: Bedbugs and infectious diseases. Clin Infect Dis. 2011, 52:200-10. 10.1093/cid/ciq102

16. Goddard J, Varela-Stokes A, Smith W, Edwards KT: Artificial infection of the bed bug with Rickettsia parkeri . J Med Entomol. 2012, 49:922-6. 10.1603/me11282

17. Zygowicz WM: What's buggin' EMS. How to rid your rigs of a bedbug infestation . JEMS. 2012, 37:56-60.

18. Erdogan J, Martin T, Payne R: EDs trying not to let the bed bugs bite . ED Manag. 2010, 22:100-1.

19. University Hospitals, EMS Training \& Disaster Preparedness Institute. Medical control/procedures: bed bugs. Prehospital care protocol and treatment guidelines. (2019). Accessed: November 21, 2019: https://www.uhems.org/pdfs/UHEMSProtocol2019R5.pdf.

20. 2019 Top 50 Bed Bug Cities in U.S . (2019). Accessed: September 23, 2019: https://www.terminix.com/blog/whats-buzzing/top-bed-bug-cities/.

21. Sheele JM, Barrett E, Farhan O, Morris N: Analysis of bed bug (Cimex lectularius) introductions into an academic medical center. Infect Control Hosp Epidemiol. 2017, 38:623-4. 10.1017/ice.2017.13

22. Sheele JM, Barrett E, Dash D, Ridge GE: Analysis of the life stages of Cimex lectularius captured within a medical centre suggests that the true numbers of bed bug introductions are under-reported. J Hosp Infect. 2017, 97:310-12. 10.1016/j.jhin.2017.07.025 


\section{Cureus}

23. Sheele JM, Mallipeddi N, Coppolino K, Chetverikova M, Mothkur S, Caiola C: FMC Verifi traps are not effective for quantifying the burden of bed bugs in an emergency department. Am J Infect Control. 2016, 44:1078-80. 10.1016/j.ajic.2016.02.013 